\title{
Photometric variability of the Be star population with the KELT survey
}

\author{
Jonathan Labadie-Bartz ${ }^{1}$, Joshua Pepper ${ }^{1}$, S. Drew Chojnowski ${ }^{2}$ \\ and M. Virginia McSwain ${ }^{1}$ \\ ${ }^{1}$ Department of Physics, Lehigh University, 16 Memorial Drive East, Bethlehem, PA 18015, \\ USA; email: jml612@lehigh.edu \\ ${ }^{2}$ Apache Point Observatory and New Mexico State University, P.O. Box 59, Sunspot, NM \\ 88349-0059, USA
}

\begin{abstract}
We are using light curves from the KELT exoplanet transit survey (Pepper et al. 2007) to study the variability of hundreds of Be stars. Combining these light curves with simultaneous time-series spectra from the APOGEE survey (Majewski et al. 2015) provides a glimpse into how changes in the circumstellar environment are correlated to brightness variations.
\end{abstract}

Keywords. stars: emission-line, Be, stars: variables: other

Be stars are rapidly rotating B-type stars with line emission, which is attributed to a gaseous circumstellar disk. Such systems can be highly variable on timescales ranging from hours to decades. KELT photometry shows numerous cases of periodic variability, including both high and low frequency signals. We attribute high frequency signals to stellar pulsations. Low frequencies may indicate binarity, the coupling of pulsational modes, or other phenomena.

Long-term variability on the order of years is not uncommon, and indicates changes in the circumstellar disk. We detect 'outbursts', where the system brightens suddenly, then fades back towards the baseline brightness, and are understood to be discrete events where material is flung from the star into the circumstellar environment, forming a disk. We find high-frequency signals in $24 \%$, low-frequency signals in $38 \%$, long-term variation in $37 \%$, and outbursts in $36 \%$ of our sample. See Labadie-Bartz et al. 2017 for more information.

Fig. 1 shows an outburst, as seen in KELT photometry, and two spectra of the Bracket 11 line, as seen by APOGEE. The spectrum taken prior to the outburst shows no sign of a disk, but after the outburst the spectrum shows a clear disk signature. This example supports the idea of photometric outbursts corresponding to the injection of stellar material into the circumstellar environment.
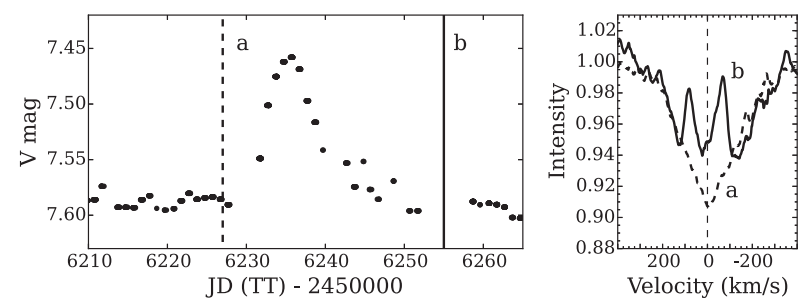

Figure 1. Left: KELT light curve showing an outburst. The 2 vertical lines show the epochs of the APOGEE observations. Right: APOGEE spectra showing the creation of a disk.

\section{References}

Labadie-Bartz, et al. 2017, 2017, AJ, 153, 252

Majewski, et al. 2015, arXiv, 1509.05420

Pepper, et al. 2007, PASP, 119, 923 